
\title{
28 Research Square \\ PTPROt Enhances the Sensitivity of Multiple Myeloma to BP-1-102 and Bortezomib
}

\section{Zixuan Li}

Soochow University

\section{Yong Liu}

Li Huili Hospital, Ningbo Medical Center

\section{Yan Xie}

Soochow University

Lei Yuan

Shenzhen University

\section{Gangli An}

Soochow University

\section{Chengcheng Fu}

Soochow University

Lin Yang ( $\nabla$ yanglin@suda.edu.cn )

Soochow University

\section{Research Article}

Keywords: PTPROt, bortezomib, STAT3, BP-1-102, multiple myeloma

Posted Date: January 28th, 2022

DOI: https://doi.org/10.21203/rs.3.rs-1259775/v1

License: (c) (i) This work is licensed under a Creative Commons Attribution 4.0 International License.

Read Full License 


\section{Abstract}

\section{Background}

Multiple myeloma $(\mathrm{MM})$ is an incurable plasma cell malignancy for which novel treatment options are urgently required. STAT3 overexpression is associated with poor prognosis of MM and could enhance chemotherapy resistance of MM cells. Therefore, targeting the expression and/or activity of STAT3 may represent a potential treatment strategy for MM.

\section{Methods and results}

This study aimed to examine the effects of protein tyrosine phosphatase receptor type 0 (PTPROt) overexpression and BP-1-102, an inhibitor of STAT3, on MM cell growth and survival. The results showed that PTPROt overexpression suppressed MM cell progression by inhibiting STAT3 phosphorylation, and that BP-1-102 inhibited the proliferation, migration, and invasion of MM cell lines in a dose-dependent manner. We also found that BP-1-102 hampered STAT3 phosphorylation and induced apoptosis in vitro, and inhibited the growth of $\mathrm{H} 929$ and MM1S xenografts in vivo. Furthermore, the combination of BP-1102 and bortezomib (BTZ) was more efficacious at killing MM cells than BP-1-102 or BTZ alone in vitro and in vivo. Notably, PTPROt also enhanced the sensitivities of multiple myeloma cell lines, primary myeloma cells, and H929-PTPROt xenograft tumors to BP-1-102 and BTZ treatments.

\section{Conclusions}

The findings of the current study strongly indicated that STAT3 could be a promising therapeutic target for the treatment of MM.

\section{Introduction}

Multiple myeloma ( $\mathrm{MM})$ is a clonal malignant disease characterized by abnormal plasma cell proliferation, and the second most common hematologic malignancy (1). Although the curative effects of proteasome inhibitors, such as bortezomib (BTZ), have demonstrated considerable progress, MM is still characterized by a high relapse rate in the terminal stage, with gradual acquisition of resistance to existing treatment options (2-4). With improved treatment options and combined myeloablative chemotherapy and autologous stem cell transplantation, the median survival time now exceeds 6 years. Despite drastic changes in treatment strategy, myeloma remains largely incurable (5). Therefore, effective therapies that either cure MM or provide persistent disease control with symptomatic relief are urgently needed.

The mammalian Signal Transducer and Activator of Transcription (STAT) family mediates various intracellular signaling pathways (6). STAT3 regulates cellular immunity as well as cell proliferation, apoptosis and differentiation (7-8). Persistent STAT3 hyperactivation (predominantly by phosphorylation) is a common event in MM cells. The activation of this protein has attracted increasing 
attention in MM research regardless of whether it directly leads to the occurrence of $\mathrm{MM}$ or is a physiological phenomenon without any biological significance (9). Therefore, this finding strongly indicated that STAT3 activation was closely linked to the occurrence of MM, and that STAT3 may be a primary therapeutic target for MM treatment.

Protein tyrosine phosphatase receptor type 0 (PTPROt) is a transmembrane protein that is mainly expressed in B lymphocytes and macrophages (10). PTPROt affects the occurrence and progression of liver cancer by regulating STAT3 phosphorylation (11). However, it remains unclear whether PTPROt affects MM cell proliferation, differentiation, apoptosis, and chemotherapy resistance by regulating the STAT3 pathway. Prior evidence showed that STAT3 may be involved in the development of resistance to conventional chemotherapy drugs (12-13); therefore, blocking STAT3 hyperactivation may reverse drug resistance during chemotherapy. BP-1-102, an inhibitor of STAT3, has been evaluated for antitumor effects in humans in vitro and in vivo (14-16); however, its role in MM has not been reported. Here, we show that PTPROt enhances the sensitivity of MM to BP-1-102, alone or in combination with bortezomib, by downregulating STAT3 activity.

\section{Methods}

\section{Reagents and antibodies}

BP-1-102 and BTZ were obtained from Selleck Chemical and stored at $-20^{\circ} \mathrm{C}$ (Selleckchem, Houston, TX). STAT3 (1:1000,), phosphorylated STAT3 (p-STAT3) (Y705; 1:1000), C-MYC (1:1000), BCL-2 (1:1000), cleaved caspase3 (1:1000), cleaved caspase8 (1:1000), cleaved caspase9 (1:1000), and MCL-1 (1:1000) were purchased from Cell Signaling Technology (Cell Signaling Technology, Inc., Danvers, MA). $\beta$-Actin (1:1000) was purchased from ProteinTech Group, Inc. Rabbit IgG HRP-conjugated antibody (1:1000) was purchased from R\&D Systems (Minneapolis, MN). Goat anti-mouse IgG/HRP (1:5000) was purchased from Solarbio (China).

\section{Cell culture}

U266, H929, and MM1S cell lines were cultured with RPMI-1640 medium with $10 \%$ fetal bovine serum and $1 \%$ penicillin/streptomycin (Thermo Fisher Scientific, Waltham, MA) in an incubator at $37^{\circ} \mathrm{C}$ in a $5 \% \mathrm{CO}_{2}$ environment.

\section{Cell viability assay}

The cell lines and primary MM cells $\left(1 \times 10^{4}\right.$ cells) were seeded in $96-$ well plates and exposed to different concentrations of BP-1-102 $(2,4,6$, and $10 \mu \mathrm{M})$ or BTZ (4 or 5nM). BP-1-102 and BTZ were dissolved in DMSO and the maximum final concentration of DMSO in the cell-culture medium was $\leq 0.1 \%$. After a 24 hour incubation period at $37^{\circ} \mathrm{C}$, the cell viability was determined using the Cell Counting Kit-8 (CCK8; Dojindo Molecular Technologies, China).

\section{Flow cytometry}


The cells, at a density of $1 \times 10^{5}$, were seeded into 24 -well plates and incubated overnight. Thereafter, the cells were treated with BP-1-102 $(2,4,6$, and $10 \mu \mathrm{M})$ or BTZ $(4$ or $5 \mathrm{nM})$ for $24 \mathrm{~h}$. The maximum final concentration of DMSO in the cell-culture medium was $\leq 0.1 \%$. Annexin V-Fluorescein Isothiocyanate (APC)/7-AAD Detection Kit (MULTI SCIENCES, China) was used to detect cell apoptosis within one hour by a Beckman Coulter (Gallios, Beckman).

Cell proliferation was detected using CSFE (Invitrogen-Life Technologies). CFSE was stored at $10 \mathrm{mM}$ in $90 \mu \mathrm{L}$ of anhydrous DMSO. The cells were incubated with CFSE $(1: 1,000)$ for $30 \mathrm{~min}$ at $37^{\circ} \mathrm{C}$ in the dark.

The cells (U266, H929, and MM1S) were seeded in 24-well plates and incubated overnight. Thereafter, the cells were exposed to different concentrations of BP-1-102 $(2,4,6$, and $10 \mu \mathrm{M})$ or BTZ (4 or $5 \mathrm{nM})$ for $24 \mathrm{~h}$, and the samples were analyzed by flow cytometry (Gallios, Beckman).

\section{Migration and Invasion assay}

Cells, at a density of $1 \times 10^{5}$, were exposed to BTZ (5nM), BP-1-102 $(6 \mu \mathrm{M})$, or DMSO in 300 $\mu \mathrm{L}$ serum-free RPMI-1640 medium and seeded into the upper chambers of a transwell chamber (Corning Incorporated, USA). The chambers were either coated with Matrigel or left uncoated. Thereafter, $600 \mu \mathrm{L}$ RPMI-1640 medium with $10 \%$ fetal bovine serum was added to the lower chambers. After a 24 -hour incubation period, the cells migrated through the membrane to the lower chamber. After removing dead cells, the cells in the lower chamber were counted using a cell counter.

\section{Western blotting analysis}

The cells were harvested, rinsed twice with ice-cold phosphate-buffered saline, and homogenized using NE-PERTM Nuclear and Cytoplasmic Extraction Reagent (Thermo Fisher Scientific). Protein concentrations were determined using a BCA kit (Beyotime Biotechnology). Total proteins from each sample were separated by $10 \%$ SDS-PAGE at a constant current. The proteins were transferred to nitrocellulose membranes and incubated with Tris-buffered saline containing 0.1\% Tween-20 (TBST) and $5 \%$ nonfat dry milk at $4^{\circ} \mathrm{C}$ overnight in the presence of antibodies against STAT3, p-STAT3, MCL-1, BCL-2, C-MYC, cleaved caspase $3 / 8 / 9$, and $\beta$-actin. The membranes were washed and incubated with HRPconjugated secondary antibody in TBST containing $5 \%$ nonfat dry milk for one hour at room temperature. Immunoreactivity was detected using Enhanced Chemiluminescent Substrate (PerkinEliner,inc.USA).

\section{Murine models}

NSG female mice were purchased from BIOCYTOGEN (China). Myeloma xenograft tumors were generated by subcutaneous injection of $1 \times 10^{6} \mathrm{H} 929,2 \times 10^{6}$ MM1S, or $3 \times 10^{6} \mathrm{H} 929$-PTPROt cells resuspended in Matrigel (BD Biosciences) into the right flank of the NSG female mice. The mice were intraperitoneally administered BTZ $(0.5 \mathrm{mg} / \mathrm{kg})$, in $10 \mathrm{mg} / \mathrm{mL}$ mannitol in saline twice weekly, and BP-1$102(6 \mathrm{mg} / \mathrm{kg})$ orally every day for 2 weeks. Control group: the group who received PBS-only. BP-1-102treated group: the group who received BP-1-102-only. BTZ-treated group: the group who received BTZonly. BP-1-102+BTZ group: the group who received the combination of BP-1-102 and BTZ. The tumor volume was measured every three days and estimated using the formula (length $\times$ width2)/2. 


\section{Quantitative real-time PCR}

Total RNA was extracted using an RNA isolation reagent kit (Omega Bio-tek, Inc). cDNA was generated by reverse transcription of total RNA $(0.1-0.5 \mu \mathrm{g})$ using random primers and a Reverse Transcription kit (Thermo Fisher Scientific) in a total reaction volume of $20 \mu \mathrm{L}$.

Quantitative PCR was performed using assays specific for PTPROt (ID, HS00243097_m1) or GAPDH (ID, Hs99999905_m1, endogenous control), TaqMan Universal PCR Master Mix (Applied Biosystems), and a SYBR PCR Array kit (Thermo Fisher Scientific). The primers used were as follows:

\begin{tabular}{|lll|}
\hline Gene & Forward Primer & Reverse Primer \\
\hline 3-actin & TTCTACAATGAGCTGCGTGTG & GGGGTGTTGAAGGTCTCAAA \\
\hline STAT3 & GGAGGAGTTGCAGCAAAAAG & TGTGTTTGTGCCCAGAATGT \\
\hline MCL-1 & CTTACGACGGGTTGGG & GGTTCGATGCAGCTTTCTTGG \\
\hline C-MYC & AAACACAAACTTGAACAGCTAC & ATTTGAGGCAGTTTACATTATGG \\
\hline cyclin D1 & CCGTCCATGCGGAAGATC & GAAGACCTCCTCCTCGCACT \\
\hline BCL-XL & CAGAGCTTTGAACAGGTAG & GCTCTCGGGTGCTGTATTG \\
\hline BCL-2 & GATTGTGGCCTTCTTTGAG & CAAACTGAGCAGAGTCTTC \\
\hline PTPROt & AGTTCGAGGAGCTGAAGTTAATCGG & GGCAGGATGTTGGTGTATCTGTTCT \\
\hline
\end{tabular}

Reactions were performed in triplicate using the Q6 Sequence Detection System (Applied Biosystems). Quantification was determined by delta-delta Ct method, and transcript levels of target genes were normalized with $\beta$-actin.

\section{Primary patient samples}

Primary myeloma cells were obtained from the bone marrow of patients diagnosed with MM. Bone marrow plasma cells of patients were sorted using StraightFrom Whole Blood CD138 MicroBeads (Miltenyi Biotec).

\section{Statistical analysis}

Statistical analyses were performed using Student's t-test (GraphPad Prism 6.01, GraphPad, Inc., La Jolla, CA, USA). A P value $<0.05$ was considered statistically significant.

\section{Results}

\section{PTPROt suppressed MM cell proliferation by inhibiting STAT3 phosphorylation}


First, we sorted myeloma cells from newly diagnosed multiple myeloma (NDMM) and relapsed drugresistant multiple myeloma (RRMM) patients and detected the expression of PTPROt using qPCR. The results showed that PTPROt expression was higher in NDMM patients than in RRMM patients (Fig. 1A). This finding indicated that patients with low PTPROt expression had a higher tumor burden and vice versa. Previous studies reported that PTPROt inhibits liver cancer cell growth through the STAT3 signaling pathway (17-18). We wanted to investigate if PTPROt affected the proliferation of myeloma cells by regulating the STAT3 signaling pathway. Therefore, we constructed U266-PTPROt, H929-PTPROt, and MM1S-PTPROt cell lines, that overexpressed PTPROt (Fig. 1B), and used qPCR to analyze STAT3, MCL-1, BCL-XL, BCL-2, C-MYC, and D-cyclin mRNA levels in MM cell lines (Fig. 1C). Compared with U266, H929, MM1S, U266-GFP, H929-GFP and MM1S-GFP cells; U266-PTPROt, H929-PTPROt and MM1S-PTPROt cells had lower expression levels of BCL-2, BCL-XL, D-cyclin, and C-MYC. However, the expression of STAT3 did not change significantly. Western blot results also showed that the expression levels of cleaved caspase9, cleaved caspase 8 and cleaved caspase 3 increased while the expression levels of C-MYC, MCL-1, p-STAT3 (Y705), BCL-2 and SOCS3 decreased; however, the expression of STAT3 did not change in U266-PTPROt, H929-PTPROt and MM1S-PTPROt cells as compared to U266, H929, MM1S, U266-GFP, H929-GFP and MM1S-GFP cells (Fig. 1D). These findings indicated that PTPROt inhibited cell proliferation and promotes cell death by hampering STAT3 phosphorylation.

\section{BP-1-102 alone, or in combination with BTZ, promoted myeloma cell apoptosis by inhibiting STAT3 phosphorylation}

PTPROt inhibits the growth of myeloma cells by inhibiting STAT3 phosphorylation. Therefore, we investigated whether the inhibition of STAT3 signaling could also inhibit the growth of myeloma cells using BP-1-102 in combination with BTZ to treat MM cells. BP-1-102 alone or combined with BTZ upregulated cleaved caspase3 expression, downregulated BCL-2 and MCL-1 expression, and inhibited p-

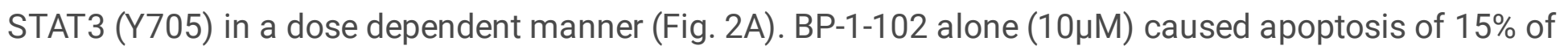
U266 cells (Fig. 2B), 90\% of H929 cells (Fig. 2C), and 55\% of MM1S cells (Fig. 2D); however, in combination with BTZ, these apoptosis percentages increased to $18 \%, 95 \%$, and $70 \%$, respectively (Fig. 2B-D). These findings demonstrated that BP-1-102 alone or in combination with BTZ induced myeloma cell apoptosis by inhibiting the phosphorylation of STAT3, which indicates that expression and/or activity blockade of STAT3 may induce myeloma cell apoptosis.

\section{BP-1-102 alone or in combination with BTZ inhibited the growth of myeloma cells}

The U266, H929, and MM1S cells were then exposed to different concentrations of BP-1-102 and BTZ to detect their effects on the proliferation of the myeloma cells by the CCK8 assay. Compared with the rate of proliferation in the control group, BP-1-102 dose-dependently inhibited the proliferation of U266 (left panel), H929 (middle panel), and MM1S cells (right panel) (Fig. 3A). Furthermore, we used CFSE to detect the proliferation of U266, H929, and MM1S cells and found that cell proliferation was dependent on the 
concentration of BP-1-102. Compared with BP-1-102 alone, the combination of BP-1-102 and BTZ better inhibited cell proliferation (Fig. 3B-D). Transwell assays were performed to assess the effects of BTZ and BP-1-102 on the migration and invasion of myeloma cells. The number of penetrating cells was remarkably lower in the BP-1-102-treated group $(6 \mu \mathrm{M})$ than in the control group (untreated) (Fig. 3E-F), and the number of migratory and invasive cells in the BP-1-102 and BTZ treated groups was the lowest. These data indicated that BP-1-102 alone or in combination with BTZ inhibited the proliferation, migration, and invasion of myeloma cells. The results suggest that expression and/or activity blockade of STAT3 may inhibit the growth of myeloma cells.

\section{Combined treatment with BP-1-102 and BTZ inhibited tumor growth}

To determine whether BP-1-102 alone, or in combination with BTZ, inhibits the growth of myeloma cells in vivo, we selected H929 cells in the logarithmic growth phase and inoculated, at a density of $1 \times 10^{6}$, subcutaneously into the outer right limb of mice. After six days, BP-1-102 was orally administered daily, and BTZ was intravenously injected into the tail vein twice per week (Fig. 4A). After 15 days of treatment, the mice in the treatment and control groups were sacrificed, and the grossly visible tumors were removed (Fig. 4B). The tumor weights and volumes in each group are presented in Fig. 4C-D. The results revealed that the tumor weight and volume were smaller in the BTZ and BP-1-102 groups than in the PBS group. Furthermore, the tumor volume and weight were smaller in the combined treatment group than in the BP1-102 group or BTZ group. To confirm that BP-1-102 alone, or in combination with BTZ, inhibits tumor cell growth, we constructed a mouse xenograft model using MM1S cells. MM1S cells, at a density of $2 \times 10^{6}$ cells, were inoculated subcutaneously into the NSG mice. BP-1-102 was orally administered daily, and BTZ was intravenously injected twice weekly (Fig. 3E). The tumor weight and volume were significantly larger in the PBS group than in the BP-1-102, BTZ, and BP-1-102+BTZ groups. Importantly, there was only one tumor in the BP-1-102+BTZ group (Fig. 3F-H). These results showed that BP-1-102, alone or in combination with BTZ, exerted a strong inhibitory effect on MM1S cells in vivo and inhibited the growth of MM cells in vivo and in vitro.

\section{PTPROt enhanced the sensitivity of MM to BP-1-102 and BTZ}

To examine whether PTPROt mediates the therapeutic effect of BP-1-102 alone, or in combination with bortezomib, on MM, these two drugs were used to treat myeloma cell lines (U266, U266-GFP, U266PTPROt; H929, H929-GFP, H929-PTPROt; MM1S, MM1S-GFP, MM1S-PTPROt). Treatment with BP-1-102 alone (10um) or in combination with bortezomib reduced the number of myeloma cells overexpressing PTPROt (Fig. 5A). PTPROt expression was upregulated in myeloma cells of NDMM patients, whereas it was downregulated in myeloma cells of RRMM patients. Therefore, we used CSFE to detect the proliferation of primary myeloma cells. When exposed to BP-1-102 $(6 \mu \mathrm{M})$ for $24 \mathrm{~h}$, the average fluorescence intensity of CSFE of myeloma cells from RRMM patients and NDMM patients decreased by 4000 and $7000 \mathrm{MFI}$, respectively (Fig. 5B-C). This finding indicated that PTPROt enhanced the sensitivity 
of myeloma cells to BP-1-102 and BTZ. Next, to determine whether PTPROt augments the sensitivity of myeloma cells to BP-1-102 and BTZ in vivo, we constructed an H929-PTPROt mouse model. Briefly, H929PTPROt cells, at a density of $3 \times 10^{6}$, were injected subcutaneously into NSG mice. After 21 days, BP-1-102 was orally administered daily, whereas BTZ was injected twice a week via the tail vein (Fig. 5D). The tumor volume was measured every three days (Fig. 5E). After 15 days, the tumor volume and weight were greater in the PBS group than in the BP-1-102 and BTZ groups. The tumor volume and weight were smaller in the BP-1-102+BTZ group than in the BP-1-102 group or BTZ group (Fig. 5E-G). Further, the tumor volume, weight, and growth rate were lower in the H929-PTPROt group than in the H929 xenograft tumors group (Fig. 4E-H). These results proved that PTPROt enhanced the sensitivity of multiple myeloma cells to BP-1-102 and BTZ in vivo and in vitro. Together, our findings indicated that STAT3 could be a promising therapeutic target for MM.

\section{Discussion}

The STAT3 signaling pathway plays an important role in cell proliferation, apoptosis, and invasion (7-8, 19), and Constitutive STAT3 activation leads to the occurrence of myeloma $(9,19)$. The genetic suppression of dominant chromosome function and RNA interference showed that a decrease in STAT3 activity was associated with a decrease in the activity of MM cells. This phenomenon strongly implies that there is a close relationship between STAT3 activation and the occurrence of MM (20). Consequently, expression and/or activity blockade of STAT3 may be a potential treatment strategy for MM (14). Our experiments proved that PTPROt with BP-1-102 alone, or in combination with BTZ, inhibited the proliferation of myeloma cells by inhibiting the phosphorylation of STAT3.

Previous studies demonstrated that PTPROt suppressed the growth of liver cancer cells by inhibiting STAT3 phosphorylation (17-18). In our present study, the STAT3 phosphorylation decreased in myeloma cells overexpressing PTPROt, causing a decreased expression of BCL-1, MCL-1, and C-MYC. There was also a concurrent increase in the expression levels of cleaved caspases 3,8 , and 9 . This indicated that PTPROt inhibited the growth of myeloma cells by inhibiting the phosphorylation of STAT3. BP-1-102, which acts by direct interaction with relatively low concentrations of STAT3, has been proven to be effective in various tumors $(14-16,21)$. However, to the best of our knowledge, its role in MM has not been adequately investigated. In the present study, we found, for the first time, that BP-1-102 alone or in combination with BTZ had superior suppressive effects on cell proliferation, migration, and invasion in U266, H929, and MM1S cells. In addition, STAT3 activation and apoptosis of MM were inhibited by BP-1102 alone in a dose-dependent manner in combination with BTZ. Furthermore, BP-1-102 alone, or in combination with BTZ, inhibited the growth of H929 and MM1S tumor cells in vivo. While BP-1-102 alone inhibited MM cell growth, and the combined use of BP-1-102 and BTZ further enhanced these inhibitory effects on MM cells in vitro and in vivo.

Previous studies also showed that PTPROt may increase sensitivity to chemotherapeutic drugs (22). BP1-102 treatment exerted a better effect, in vitro, on cell expressing high levels of PTPROt than on those with low PTPROt expression levels. BP-1-102 alone, or in combination with BTZ, better inhibited the 
growth of H929-PTPROt xenograft tumors than that of $\mathrm{H} 929$ xenograft tumor cells in vivo. The limitation of this study was the fact that even when the tumor volume of $\mathrm{H} 929$ xenograft cells reached $1000 \mathrm{~mm}^{3}$, the H929-PTPROt tumor remained invisible to the naked eye. Therefore, we could not inoculate H929 and H929-PTPROt cells into the same mouse simultaneously.

Our study is the first to demonstrate that PTPROt and BP-1-102 alone, or in combination with BTZ, inhibit the proliferation of myeloma cells by inhibiting the phosphorylation of STAT3 and that PTPROt enhances the sensitivity of MM to BP-1-102 and bortezomib. These findings indicate that STAT3 may serve as a potential therapeutic target for the development of new MM therapies.

\section{Declarations}

\section{Data availability}

All data included in this study are available upon request by contacting the corresponding authors.

\section{Funding}

This study was supported by the Natural Science Foundation of China (Grant Nos. 81872431 and 31471283), the National Key R\&D Program of China (2016YFC1303403), the Priority Academic Program Development of Jiangsu Higher Education Institutions, the Collaborative Innovation Major Project (Grant No. XYXT- 2015304), the Six Talent Peaks Project in Jiangsu Province (No. SWYY-CXTD-010), and the Natural Science Foundation of the Jiangsu Higher Education Institutions of China (General Program, Grant No.19KJD320003).

\section{Acknowledgments}

U266 was a gift from PersonGen (Suzhou, China).

\section{Author contributions}

Lin Yang, Zhengzheng Fu, and Zixuan Li are responsible for the experimental design, writing, and revision of the manuscript. Yong Liu, Yan Xie, Tian Wang, Shuaiyu Tian, Kailu Zhang, and Gangli An provided help during animal and cell experiments.

\section{Author statement}

All authors declare that there is no conflict of interest. All authors have read and approved the manuscript.

\section{Competing interests}

The authors declare no potential conflicts of interest for the research, authorship, and/or publication of the present article. 


\section{Ethics approval}

Patient samples were collected from the First Affiliated Hospital of Soochow University, after obtaining a written informed consent from each patient and research ethics board approval in accordance with the Declaration of Helsinki (approval no. NSFC2018 $₫ 81872431$ ).

Mouse studies were conducted in accordance with the federal and institutional guidelines using protocols approved by the Ethics Committee of Soochow University (approval no. NSFC2018ه81872431).

\section{References}

1. Kumar SK, Callander NS, Alsina M, Atanackovic D, Biermann JS Chandler J. C, Multiple, Myeloma (2017) Version 3.2017, NCCN Clinical Practice Guidelines in Oncology. J Natl Compr Canc Netw 15(2):230-269. DOI: 10.6004/jnccn.2017.0023

2. Cook G, Zweegman S, Mateos MV, Suzan F, Moreau P (2018) A question of class: treatment options for patients with relapsed and/or refractory multiple myeloma. Crit Rev Oncol 121:74-89. DOI: 10.1016/j.critrevonc.2017.11.016

3. Nijhof IS, van de Donk NWCJ, Zweegman S, Lokhorst HM (2018) Current and new therapeutic strategies for relapsed and refractory multiple myeloma: an update. Drugs 78(1):19-37. DOI: 10.1007/s40265-017-0841-y

4. Neri P, Bahlis NJ, Paba-Prada C, Richardson $P$ (2016) Treatment of relapsed/refractory multiple myeloma. Cancer Treat Res 169:169-194. DOI: 10.1007/978-3-319-40320-5_10

5. Minnie SA, Hill GR (2020) Immunotherapy of multiple myeloma. J Clin Invest 130(4):1565-1575. DOI: $10.1172 / \mathrm{JCl} 129205$

6. Darnell JE, Kerr IM, Stark GR (1994) Jak-STAT pathways and transcriptional activation in response to IFNs and other extracellular signaling proteins. Science 264(5164):1415-1421. DOI:

10.1126/science.8197455

7. Hanlon MM, Rakovich T, Cunningham CC, Ansboro S, Veale DJ, Fearon U et al (2019) STAT3 mediates the differential effects of oncostatin M and TNFa on RA synovial fibroblast and endothelial cell function. Front Immunol; 10:2056. DOI: 10.3389/fimmu.2019.02056

8. Kaushal M, Chorawala M (2012) The JAK/STAT signaling pathway. Int J Adv Res Pharm Biosci 1(4):363-386

9. Bharti AC, Shishodia S, Reuben JM, Weber D, Alexanian R, Raj-Vadhan S et al (2004) Nuclear factorkappaB and STAT3 are constitutively active in CD138+ cells derived from multiple myeloma patients, and suppression of these transcription factors leads to apoptosis. Blood 103(8):3175-3184. DOI: 10.1182/blood-2003-06-2151

10. Wakim J, Arman E, Becker-Herman S, Kramer MP, Bakos E, Shachar I et al (2017) The PTPROt tyrosine phosphatase functions as an obligate haploinsufficient tumor suppressor in vivo in B-cell chronic lymphocytic leukemia. Oncogene 36(26):3686-3694. DOI: 10.1038/onc.2016.523 
11. Hou J, Deng L, Zhuo H, Lin Z, Chen Y, Jiang R et al (2015) PTPROt maintains T cell immunity in the microenvironment of hepatocellular carcinoma. J Mol Cell Biol 7(4):338-350. DOI:

10.1093/jmcb/mjv047

12. Chong PSY, Chng W-J, de Mel S (2019) STAT3: A Promising Therapeutic Target in Multiple Myeloma. Cancers (Basel) 11(5):731. DOI: 10.3390/cancers11050731

13. Lee JH, Kim C, Sethi G, Ahn KS (2015) Brassinin inhibits STAT3 signaling pathway through modulation of PIAS-3 and SOCS-3 expression and sensitizes human lung cancer xenograft in nude mice to paclitaxel. Oncotarget 6(8):6386-6405. DOI: 10.18632/oncotarget.3443

14. Zhang X, Yue P, Page BD, Li T, Zhao W, Namanja AT et al (2012) Orally bioavailable small-molecule inhibitor of transcription factor Stat3 regresses human breast and lung cancer xenografts. Proc Natl Acad Sci USA 109(24):9623-9628. DOI: 10.1073/pnas.1121606109

15. Resetca D, Haftchenary S, Gunning PT, Wilson DJ (2014) Changes in signal transducer and activator of transcription 3 (STAT3) dynamics induced by complexation with pharmacological inhibitors of Src homology 2 (SH2) domain dimerization. J Biol Chem 289(47):32538-32547. DOI:

10.1074/jbc.M114.595454

16. Jiang X, Tang J, Wu M, Chen S, Xu Z, Wang H et al (2019) BP1102 exerts an antitumor effect on the AGS human gastric cancer cell line through modulating the STAT3 and MAPK signaling pathways. Mol Med Rep 19(4):2698-2706. DOI: 10.3892/mmr.2019.9892

17. Hou J, Xu J, Jiang R, Wang Y, Chen C, Deng L et al (2013) Estrogen-sensitive PTPRO expression represses hepatocellular carcinoma progression by control of STA T3. Hepatology 57(2):678-688. DOI: 10.1002/hep.25980

18. Motiwala T, Ghoshal K, Das A, Majumder S, Weichenhan D, Wu YZ et al (2003) Suppression of the protein tyrosine phosphatase receptor type 0 gene (PTPRO) by methylation in hepatocellular carcinomas. Oncogene 22(41):6319-6331. DOI: 10.1038/sj.onc.1206750

19. Zhang X, Yue P, Fletcher S, Zhao W, Gunning PT, Turkson J (2010) A novel small-molecule disrupts Stat3 SH2 domain-phosphotyrosine interactions and Stat3-dependent tumor processes. Biochem Pharmacol 79(10):1398-1409. DOI: 10.1016/j.bcp.2010.01.001

20. Frank DA (2003) STAT signaling in cancer: insights into pathogenesis and treatment strategies. Cancer Treat Res 115:267-291. DOI: 10.1007/0-306-48158-8_11

21. Page BD, Fletcher S, Yue P, Li Z, Zhang X, Sharmeen S et al (2011) Identification of a nonphosphorylated, cell permeable, small molecule ligand for the Stat3 SH2 domain. Bioorg Med Chem Lett 21(18):5605-5609. DOI: 10.1016/j.bmcl.2011.06.056

22. Wang H, Baladandayuthapani V, Wang Z, Lin H, Berkova Z, Davis RE et al (2017) Truncated protein tyrosine phosphatase receptor type $O$ suppresses AKT signaling through IQ motif containing GTPase activating protein 1 and confers sensitivity to bortezomib in multiple myeloma. Oncotarget 8(69):113858-113873. DOI: 10.18632/oncotarget.23017., 29371952

\section{Figures}



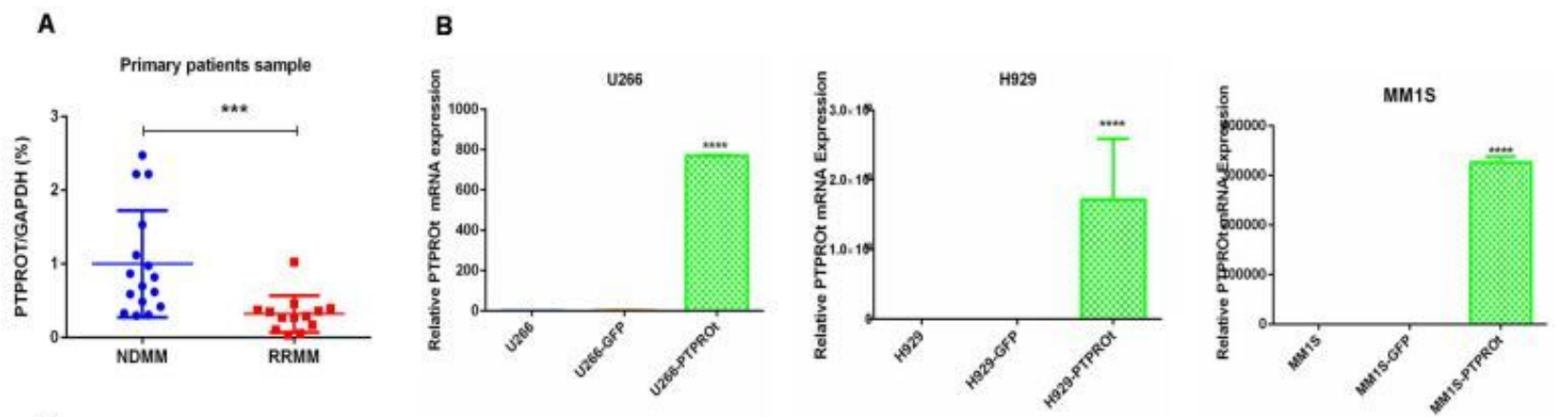

c
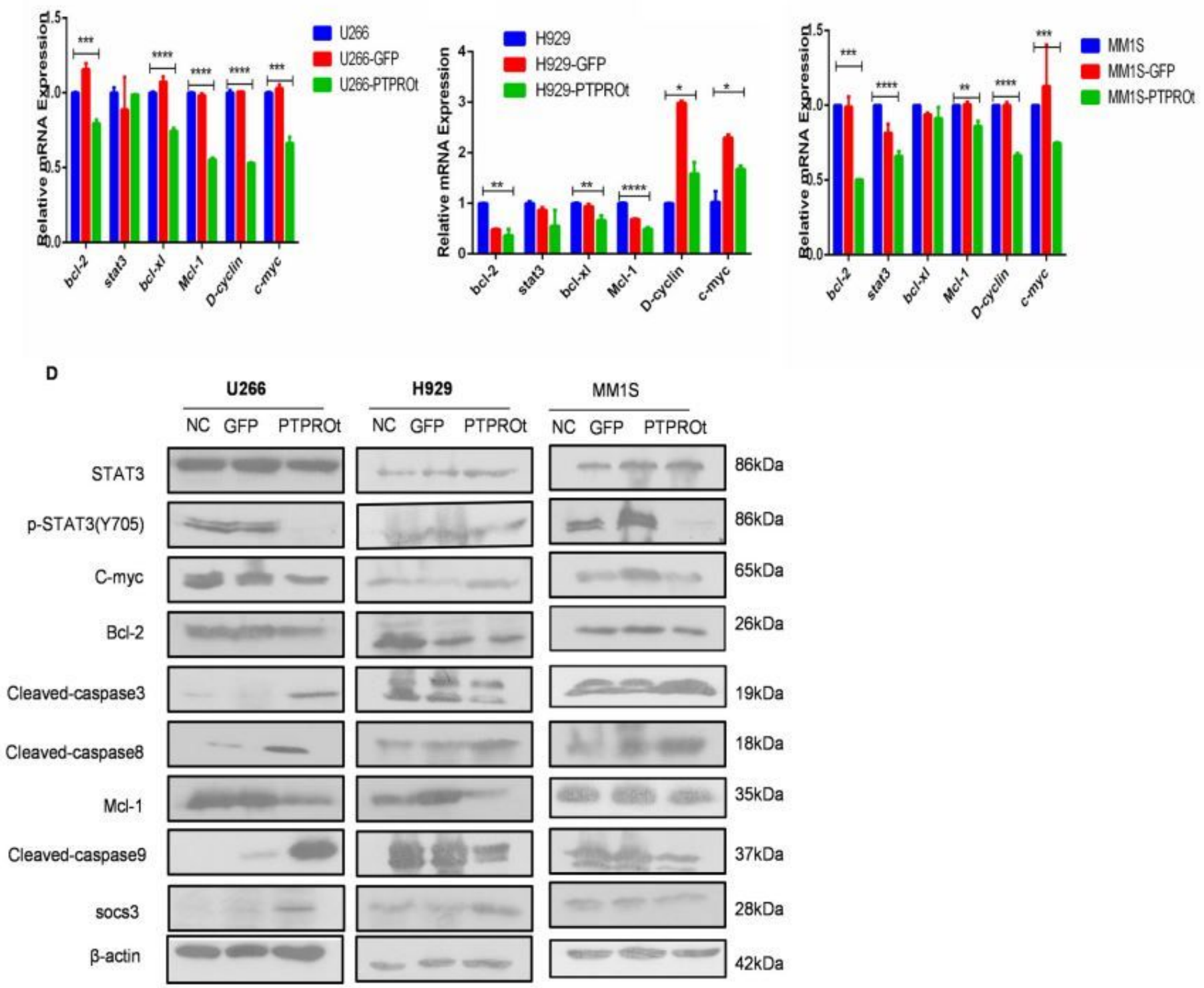

\section{Figure 1}

PTPROt inhibits myeloma cell proliferation by inhibiting STAT3 phosphorylation (A) Myeloma cells were sorted from newly diagnosed multiple myeloma (NDMM) and relapsed drug-resistant multiple myeloma (RRMM) patients. qPCR was performed to determine the expression of PTPROt in NDMM and RRMM patients using TaqMan Universal PCR Master Mix. (B) U266, H929, and MM1S cells were transfected with GFP plasmid and PTPROT plasmid lentivirus. After 24h, qPCR detected, using a SYBR PCR Array kit, 
PTPROt expression in U266 (left panel), H929 (middle panel), and MM1S (right panel) cells. (C) The mRNAs expression of STAT3, C-MYC, BCL-XL, MCL-1, and D-cyclin in U266 (left), H929 (middle), and MM1S (right) cells. (D) The protein expression of STAT3, p-STAT3(Y705), C-MYC, BCL-2, cleaved caspase3, cleaved caspase9, cleaved caspase8, MCL-1, and SOCS3. $\beta$-Actin was used as a loading control. BTZ: bortezomib.

(student's t-tests, ${ }^{\star} p<0.05,{ }^{\star \star} p<0.01$ and ${ }^{\star \star \star} p<0.001$, vs. control).

A
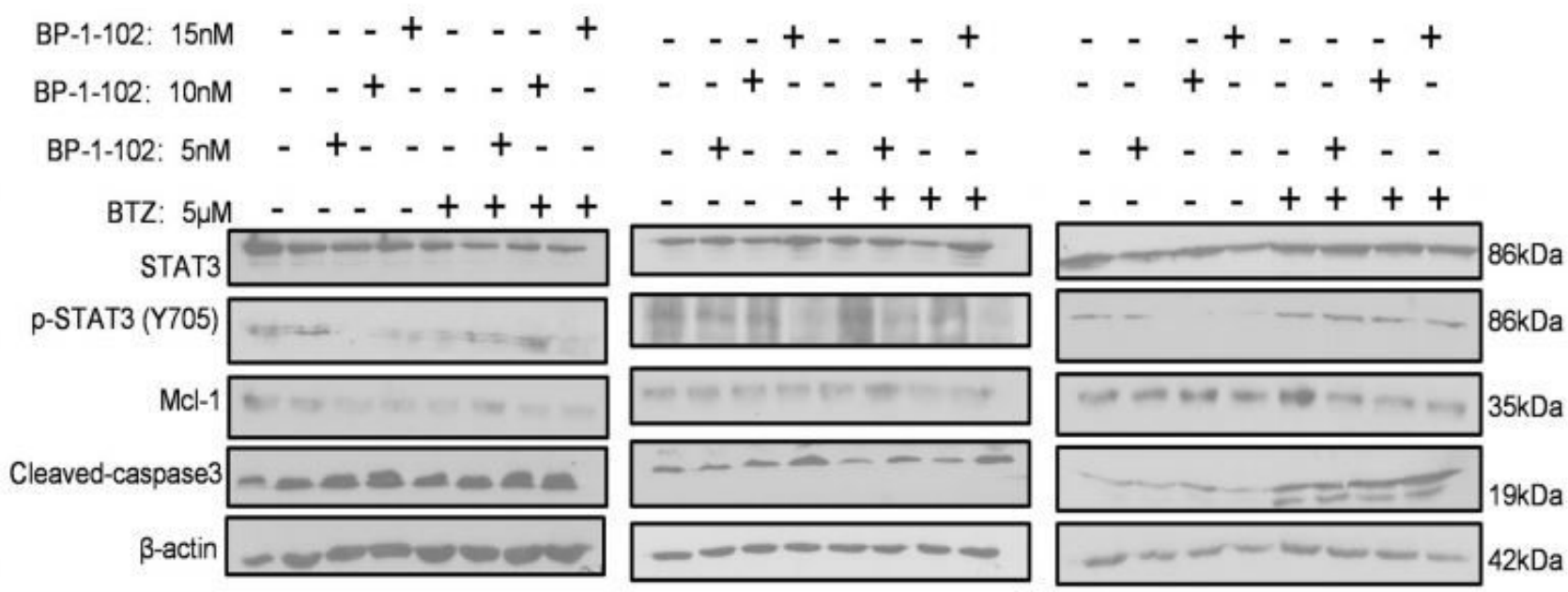

B

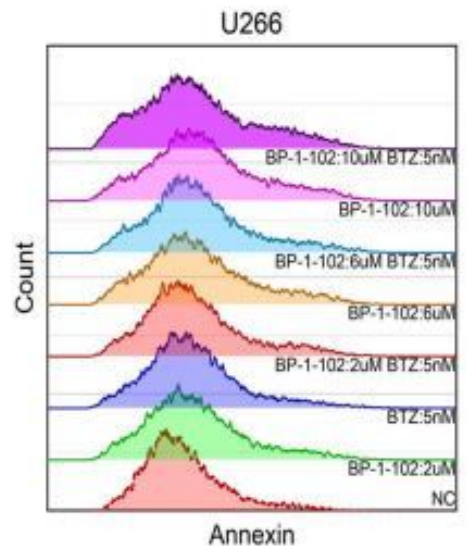

U266 BP-1-1028BTZ

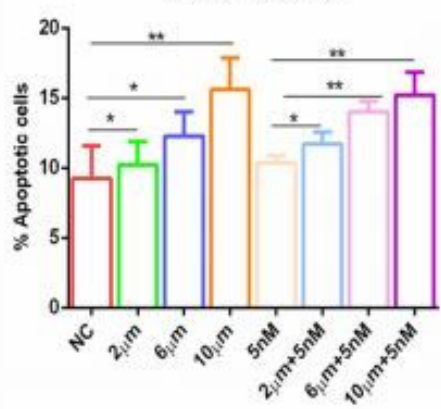

C

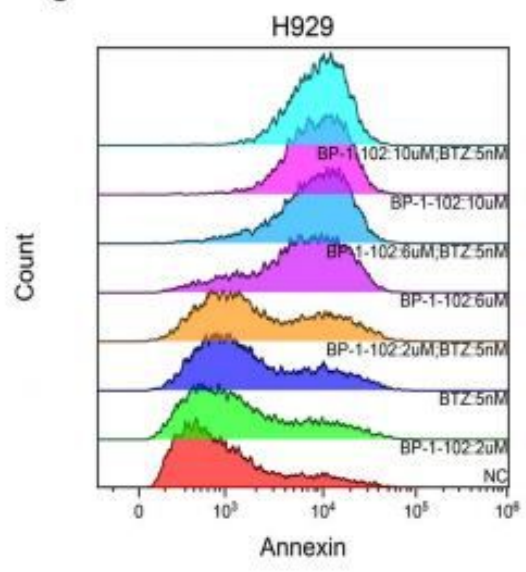

H929 BP-1-1028BTZ

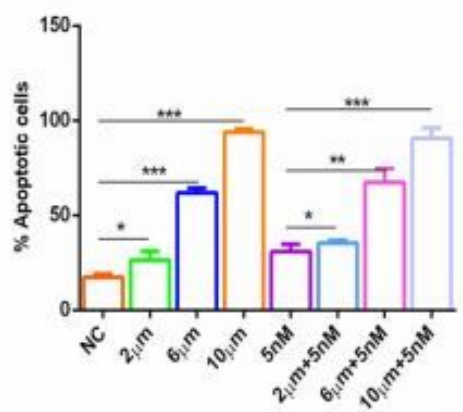

D

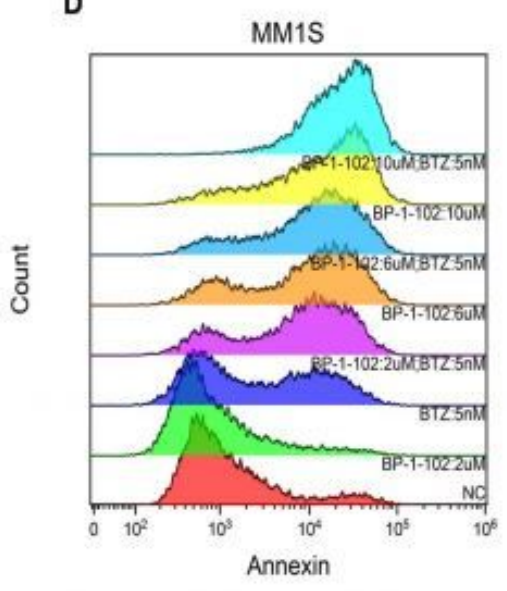

MM1S BP-1-1028BTZ

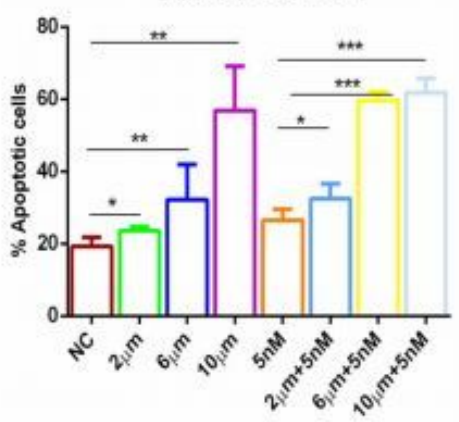


BP-1-102 alone or in combination with BTZ induces myeloma cell line apoptosis (A) Western blot analysis of STAT3, p-STAT3 (Y705), MCL-1, and cleaved caspase3 in U266, H929, and MM1S cells following exposure to different concentrations of BP-1-102 $(5,10$, or $15 \mu \mathrm{M})$ and BTZ (5nM) for $24 \mathrm{~h}$. $\beta$ Actin was used as a loading control.

(B-D) Dual annexin V and 7-AAD staining of U266, H929, and MM1S cells was analyzed using flow cytometry to evaluate the apoptosis of U266, H929, and MM1S cells after exposure to different concentrations of BP-1-102 $(2,6$, or $10 \mu \mathrm{M})$ and BTZ $(5 \mathrm{nM})$ for $24 \mathrm{~h}$. (student's t-tests, ${ }^{\star} p<0.05,{ }^{\star \star} p<0.01$ and $* \star \star p<0.001$, vs. control). BTZ: bortezomib. 
A
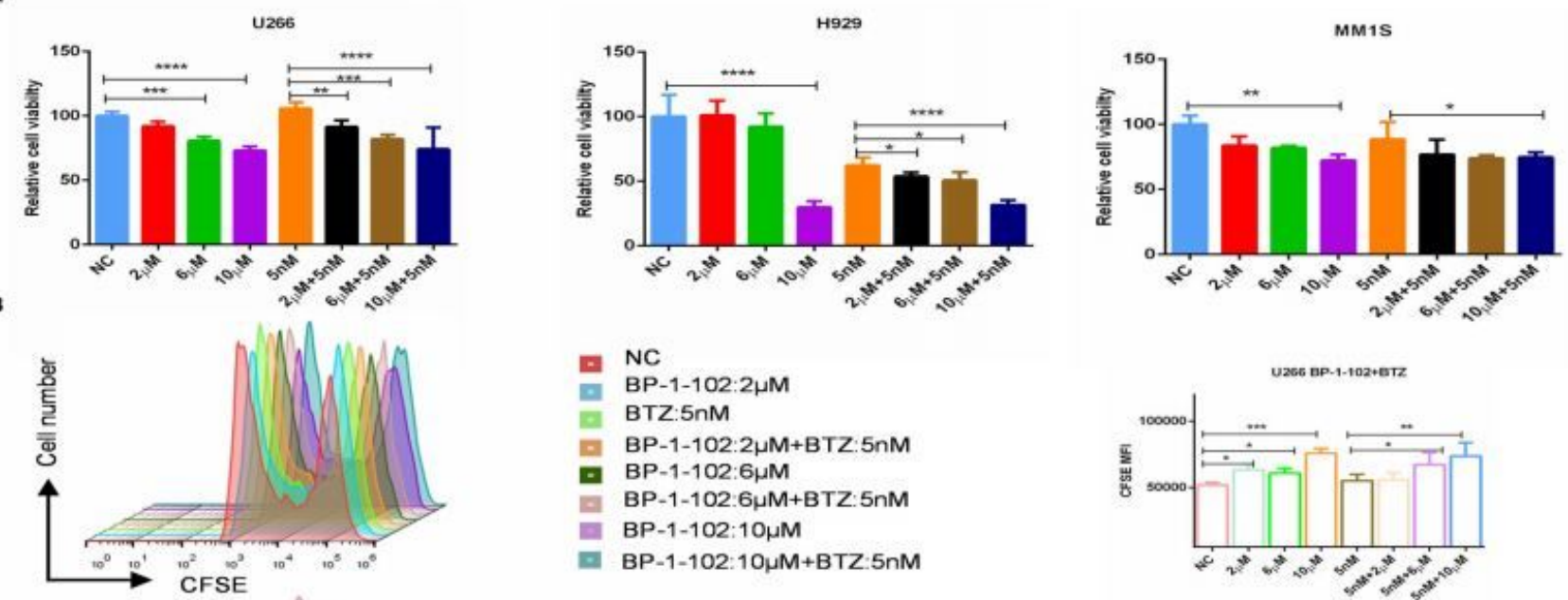

c
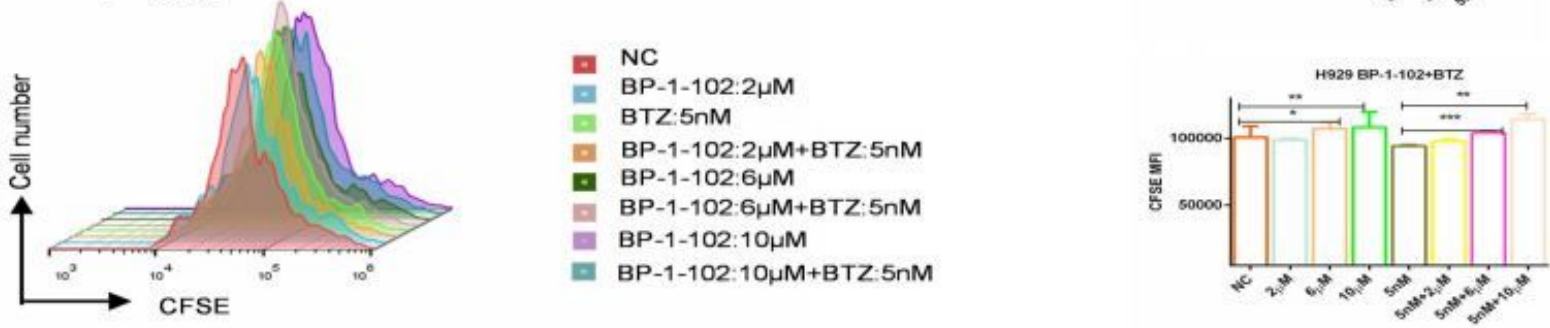

D

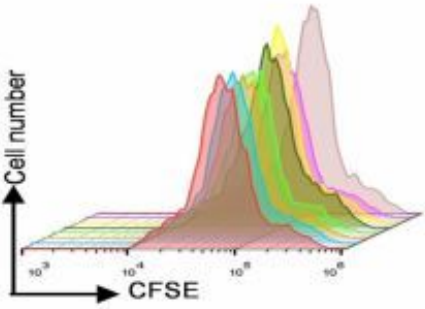

E
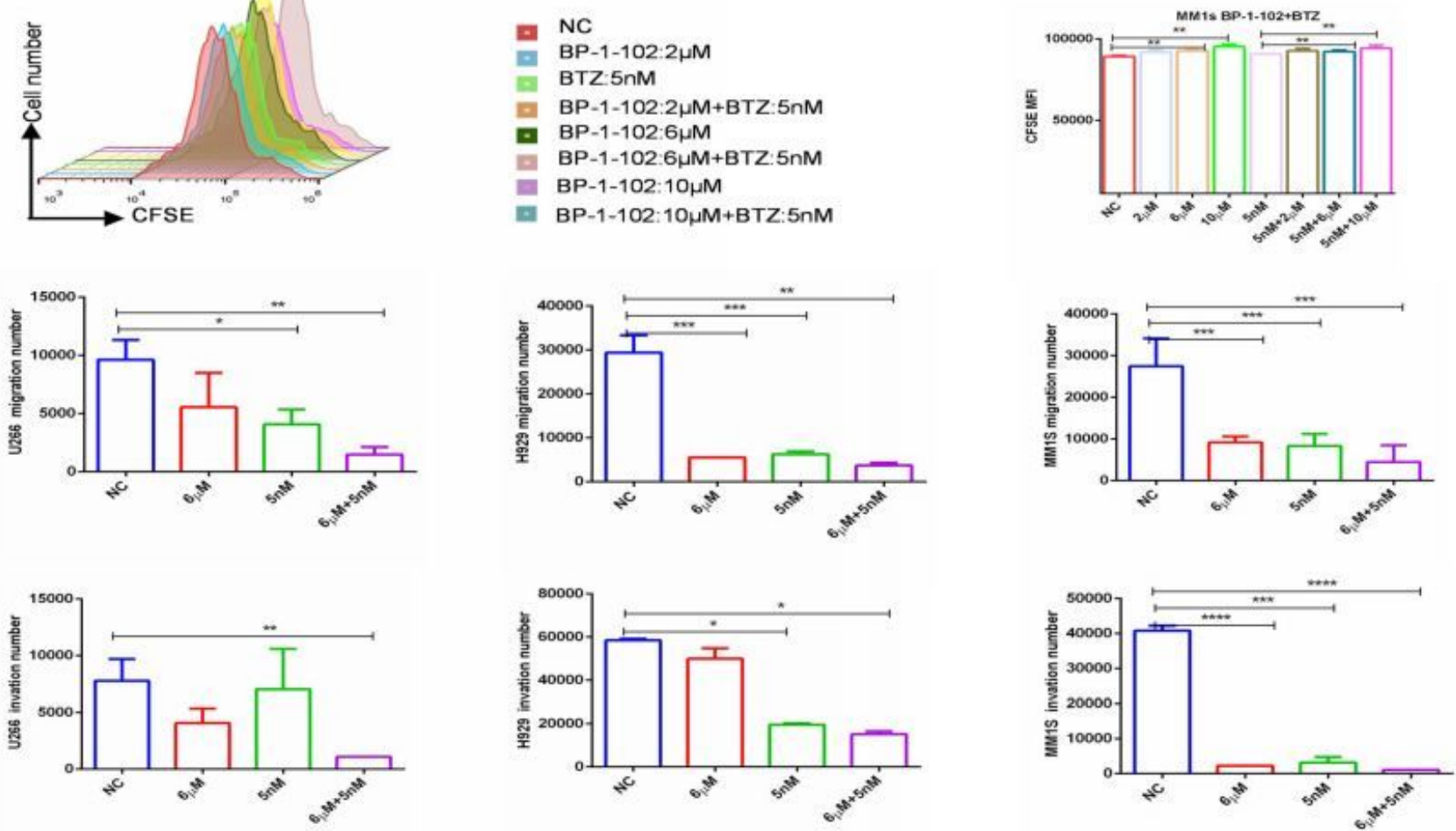

\section{Figure 3}

BP-1-102 alone or in combination with BTZ inhibits the proliferation of myeloma cells (A) Cells were treated with various concentrations of BP-1-102 $(2,6$, or $10 \mu \mathrm{M})$ and BTZ $(5 n M)$ for $24 \mathrm{~h}$. Cell activity was detected with CCK-8. (B-D) The cells were incubated with CFSE $(1: 1,000)$ for $30 \mathrm{~min}$ at $37^{\circ} \mathrm{C}$, washed thrice, and then exposed to different concentrations of BP-1-102 (2,6, or $10 \mu \mathrm{M})$ and BTZ (5nM) for $24 \mathrm{~h}$. The average fluorescence intensity of CFSE was detected using flow cytometry. (E-F) Transwell assays 
showed that BP-1-102 and BTZ significantly attenuated the migration (without Matrigel) and invasion ability (with Matrigel) of MM cells. The top compartment shows the cells after treatment with BP-1-102 (6 $\mu \mathrm{M})$ and BTZ $(5 \mathrm{nM})$ and 24-h incubation. The numbers of migrated and invading cells were quantified from three independent experiments. (student's t-tests, ${ }^{\star} p<0.05$, ${ }^{\star} p<0.01$ and ${ }^{\star \star *} p<0.001$, vs. control). BTZ: bortezomib.

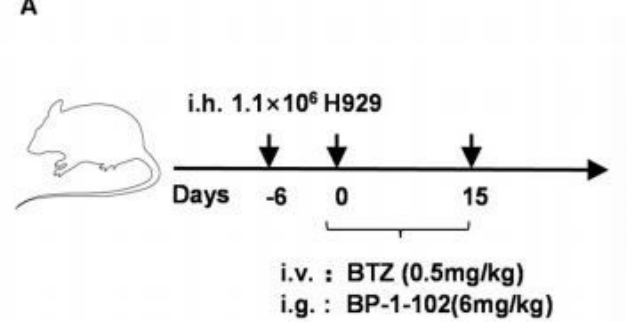

D

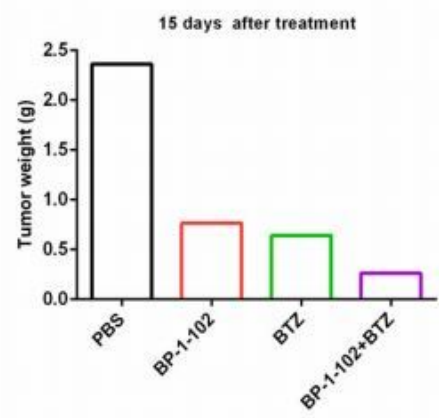

G

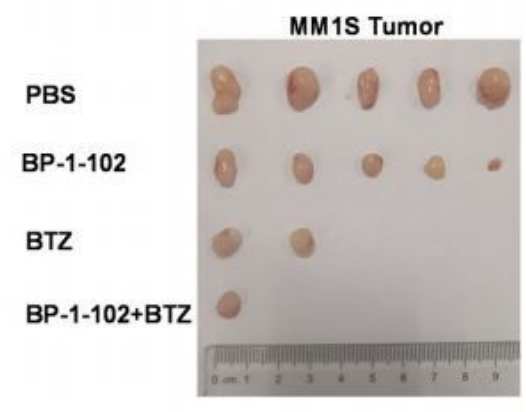

B

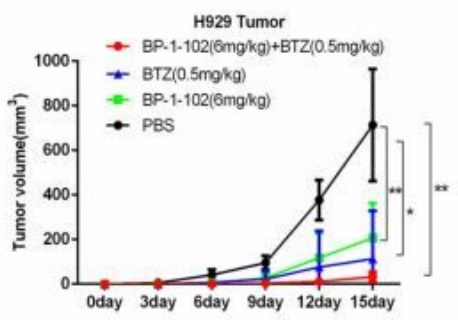

E
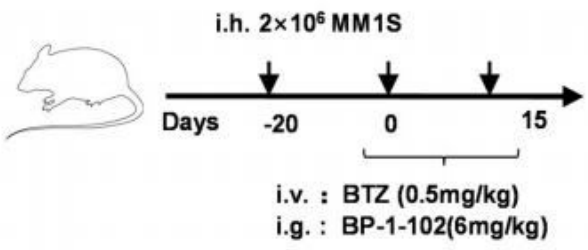

H

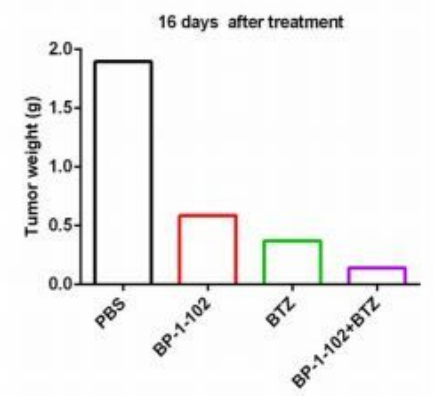

C

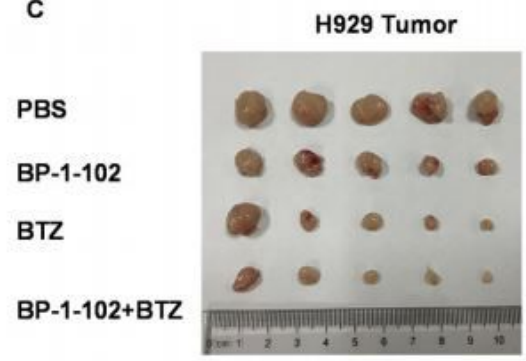

$\mathrm{F}$

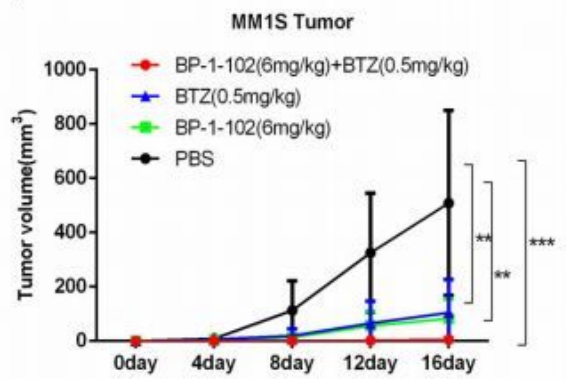

\section{Figure 4}

BP-1-102 alone or in combination with BTZ inhibits the growth of H929 and MM1S xenograft tumors (A) H929 cells $\left(1 \times 10^{6}\right)$ were injected subcutaneously into NSG mice. After $6 \mathrm{~d}, \mathrm{BP}-1-102(6 \mathrm{mg} / \mathrm{kg})$ was administered orally every day and BTZ $(0.5 \mathrm{mg} / \mathrm{kg})$ was injected intravenously twice per week via the tail vein for 15 consecutive days ( $n=5 /$ group). (B) Starting from day 0 , the tumor size was measured every three days. The tumor size was calculated using the formula (length $\times$ width $\left.{ }^{2}\right) / 2$ ( $n=5 /$ group). (C-D) The size and weight of H929 xenograft tumors in the PBS, BP-1-102, BTZ, and BP-1-102+BTZ groups after treatment for 15 days ( $n=5 /$ group). (E) MM1S cells were injected subcutaneously into NSG mice. After 20 days, BP-1-102 $(6 \mathrm{mg} / \mathrm{kg})$ was administered orally daily and BTZ $(0.5 \mathrm{mg} / \mathrm{kg})$ was intravenously 
administered twice per week for 16 days (n=5/group). (F) Volume of MM1S xenograft tumors ( $n=5 /$ group). (G-H) Tumors were harvested 16 days after treatment. The measured size and weight of the MM1S xenograft tumors in the PBS, BP-1-102, BTZ, and BP-1-102+BTZ groups are shown ( $\mathrm{n=5/group).}$ (student's t-tests, ${ }^{*} p<0.05,{ }^{* \star} p<0.01$ and ${ }^{* * *} p<0.001$, vs. control). BTZ: bortezomib.

A
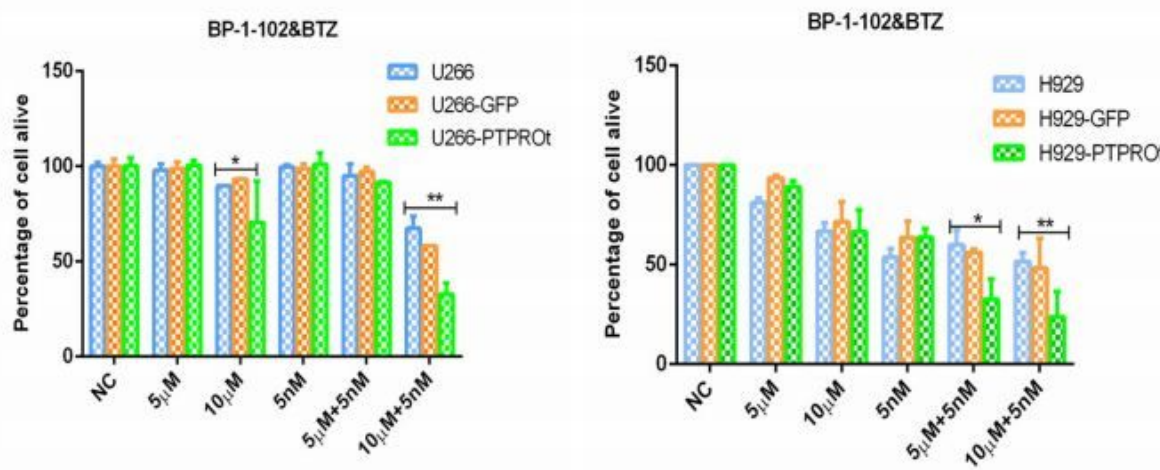

B

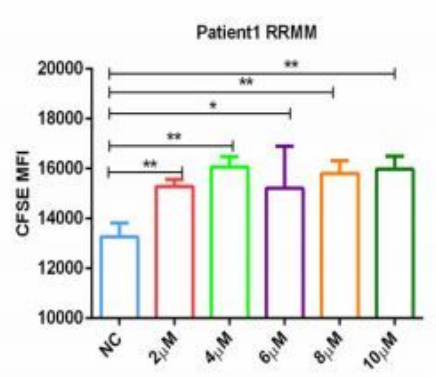

C

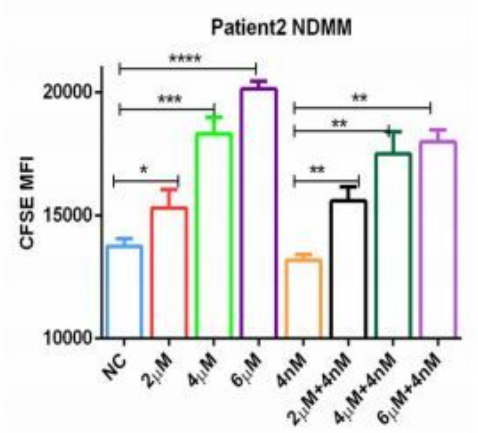

$E$

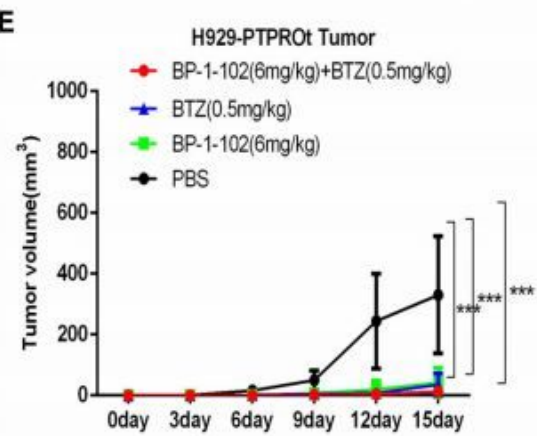

$\mathbf{F}$

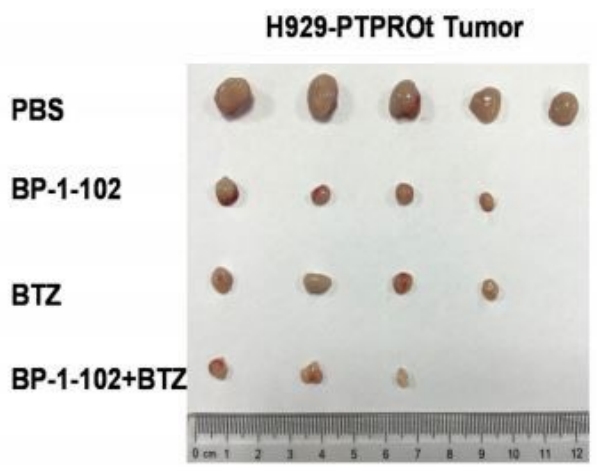

i.h. $3 \times 10^{6}$ H929-PTPROt

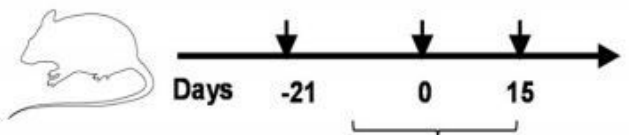

i.v. : $B T Z(0.5 \mathrm{mg} / \mathrm{kg})$

i.g. : BP-1-102(6mg/kg)

G

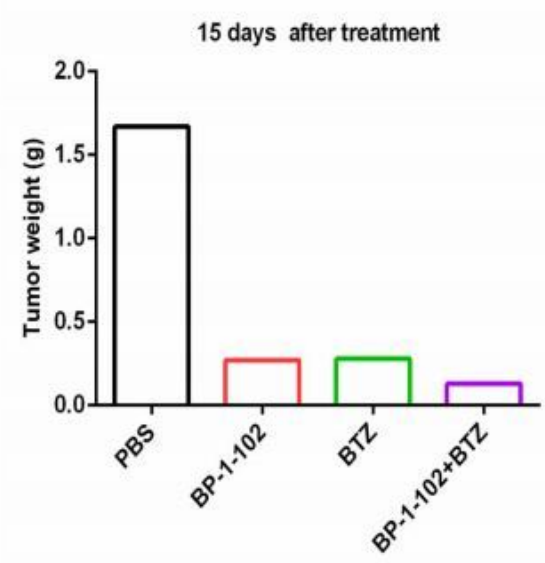

Figure 5

PTPROt increases the sensitivity of multiple myeloma to BP-1-102 and BTZ (A) U266, U266-GFP, U266PTPROt (left panel), H929, H929-GFP, H929-PTPROt (middle panel), and MM1S, MM1S-GFP, MM1SPTPROt (right panel) cells were seeded into 24-well plates and exposed to different concentrations of BP1-102 (5 or $10 \mu \mathrm{M})$ and BTZ (5nM). Flow cytometry was used to determine the proliferation and activity levels of the myeloma cell lines. (B) Primary myeloma cells from RRMM patients were exposed to 
different concentrations of BP-1-102 $(2,4,6,8$ or $10 \mu \mathrm{M})$. After $24 \mathrm{~h}$, CFSE were used to detect cell proliferation. (C) Primary myeloma cells of NDMM patients were exposed to BP-1-102 $(2,4$, or $6 \mu \mathrm{M})$ and BTZ (4nM). After $24 \mathrm{~h}$, the average CFSE fluorescence intensity was measured using flow cytometry. (D) H929-PTPROt $\left(3 \times 10^{6}\right)$ cells were injected subcutaneously into NSG mice. After 21 days, BP-1-102 (6 $\mathrm{mg} / \mathrm{kg})$ was orally administered every day and BTZ $(0.5 \mathrm{mg} / \mathrm{kg})$ was intravenously injected twice a week for 16 days ( $n=5 /$ group). (E-G) Tumors were harvested 16 days after treatment, and the volume, size, and weight of the H929-PTPROt xenograft tumors in the PBS, BP-1-102, BTZ, and BP-1-102+BTZ groups were measured ( $\mathrm{n}=5 /$ group). (student's t-tests, ${ }^{*} p<0.05$, ${ }^{*} p<0.01$ and ${ }^{* * *} p<0.001$, vs. control). NDMM: newly diagnosed multiple myeloma; RRMM: relapsed drug-resistant multiple myeloma; BTZ: bortezomib 